\title{
Molecular characterization of Ribosomal DNA (ITS2) of hard ticks in Iran: understanding the conspecificity of Dermacentor marginatus and $D$. niveus
}

\author{
Parisa Soltan-Alinejad ${ }^{1,2}$, Zahra Ramezani ${ }^{1,2}$, Hamideh Edalat ${ }^{3}$, Zakkyeh Telmadarraiy ${ }^{3}$, Farrokh Dabiri ${ }^{2}$, \\ Hassan Vatandoost ${ }^{3}$, Mohammad Ali Oshaghi ${ }^{3}$, Mehdi Mohebali ${ }^{3}$, Seyyed Javad Seyyed-Zadeh², \\ Zabihollah Zarei ${ }^{3}$, Haleh Hanifian ${ }^{2}$, Faham Faghihi ${ }^{3}$, Mandan Abolhasani ${ }^{3}$, Mulood Mohammadi Bavani², \\ Jalil Musavi ${ }^{4}$, Olle Terenius ${ }^{5}$ and Ali Reza Chavshin ${ }^{1,2^{*}}$ (B)
}

\begin{abstract}
Objectives: Hard ticks (Acari: Ixodidae) are ectoparasites of medical and veterinary importance. They are obligate blood-feeding vectors with the ability to transmit a wide variety of pathogens. Standard morphological keys are normally used for the identification of tick species. However, considering the importance of accurate species identification and the determination of bio-ecological characteristics of species, relying on morphological keys alone can be questionable. In this study, two DNA fragments (ITS2 and COI) were selected for phylogenetic evaluation of Iranian hard tick species belonging to the genera Dermacentor, Hyalomma, and Rhipicephalus.

Results: 1229 specimens of Dermacentor marginatus, D. niveus, Hyalomma anatolicum, Rhipicephalus bursa, and R. sanguineus s.l constituting 11 populations were collected from three different climatic and zoogeographical zones in Iran. Morphological studies revealed notable differences in important morphological characteristics between different populations of D. marginatus.

The results of ITS2 sequence analysis provided additional evidence which supports the conspecificity of $D$. niveus and D. marginatus. Contrary to this finding, the sequence analysis of COI and phylogeny favored the separation of the two species. Given the greater importance of $\mathrm{COI}$ in identifying and discriminating species, a possibility heterospecificity between the two species should be considered.
\end{abstract}

Keywords: Ixodidae, Molecular systematics, Dermacentor, ITS2, COI

\section{Introduction}

Hard ticks are obligate blood-feeding ectoparasites of medical and veterinary importance. They transmit a wide range of bacterial and viral diseases such as ehrlichiosis,

\footnotetext{
*Correspondence: chavshin@umsu.ac.ir; chavshin@gmail.com

${ }^{1}$ Social Determinants of Health, Research Center, Urmia University

of Medical Sciences, Urmia, Iran

Full list of author information is available at the end of the article
}

Lyme disease, Crimean Congo hemorrhagic Fever (CCHF), tularemia and anaplasmosis [1]. Considering the importance of species identification and the determination of bio-ecological characteristics of species, there is a need for thorough investigations on the taxonomic and phylogenetic relationships among hard-tick taxa. Morphological methods have been widely used for these purposes, but in some species relying solely on morphological methods may be questionable [2]. Molecular 
methods, which focus on DNA sequence differences, seems to provide a better tool for the assessment of variation within and between species [3, 4]. There is a rapid evolution of the ITS regions in the tick genome as compared to coding regions. For this reason, ITS regions have been exploited for the discrimination between closely related taxa [5]. Many molecular studies have used this region for the identification of different tick taxa [6-10]. Also, there is a high degree of hyper-variability in the second internal transcribed spacer (ITS 2) which can discriminate different populations of the same species [11, 12].

The status of the species $D$. niveus remains debatable among researchers [13]. D. marginatus complex comprises of $D$. marginatus, $D$. niveus, $D$. nuttalli, $D$. silvarum, and D. ushakovae. Previous studies have described the discrimination between these species as difficult. Some researchers argue that $D$. niveus is a synonym of $D$. daghestanicus, whereas some others believe in the heterospecificity of $D$. niveus, with $D$. daghestanicus as its junior synonym [14]. Some authors also consider both $D$. daghestanicus and $D$. niveus as valid species [15], but this opinion has not been generally accepted [2]. Moreover, molecular studies of the ITS2 fragment have suggested a conspecificity of $D$. marginatus and D. niveus was [16], but other authors still consider these two species as valid, separate species (e.g. [2]). Thus, the validation of the suggestion of conspecificity of these species awaits results from further studies.

The molecular characterization of Ixodidae ticks is of great public health importance, and the application of the ITS2 fragment for tick phylogeny remains a valuable tool for species discrimination. In the present study, 10 populations of four species belonging to three genera of Iranian hard ticks were characterized using the ITS2 fragment. Also, the possibility of the conspecificity of $D$. niveus and $D$. marginatus was examined in the present study using proper phylogenetic analysis of both ITS2 and COI fragments.

\section{Main text}

\section{Materials and methods}

\section{Study area}

Tick samples were collected from 5 provinces across Iran: Ardebil, Kerman, Tehran, Isfahan, and the Kurdistan Province (Additional file 1: Figure S1).

\section{Sample collection and identification}

Animal dwellings were visited and Ixodidae ticks were collected seasonally from the abdomen, neck, throat and the legs of hosts (sheep, cows, goats and dogs) using forceps so as not to harm the ticks. The tick specimens were placed inside a collection tube [17]. Information such as collection site, date, humidity and temperature of collection sites, and livestock owner' name were recorded for each specimen. Samples were identified to species level using standard morphological keys $[18,19]$. For the morphological differentiation of D. marginatus and D. niveus, the keys of $[13,20]$ were used.

\section{Genomic DNA extraction and $P C R$ reactions}

Samples were frozen in liquid nitrogen and homogenized. Genomic DNA was extracted from tick samples individually using AccuPrep ${ }^{\circledR}$ Genomic DNA Extraction Kit (Bioneer, South Korea), according to the manufacturer manual. The extracted DNA from each species was subjected to PCR reactions using super PCR mastermix ${ }^{\circledR}$ (Yekta Tajhiz Azma, Iran) and the primers: DITS2-F, $5^{\prime}$-GTGCGTCCGTCGACTCGTTTTGA-3' ${ }^{\prime}$ and DITS2$\mathrm{R}, 5^{\prime}$-ACGGCGGACTACGACGGAATGC-3' [12], in order to amplify the ITS2 region. The amplification condition for the ITS2 region was as follows: initial denaturation at $95^{\circ} \mathrm{C}$ for $5 \mathrm{~min}$ followed by 30 cycles of $\left[95^{\circ} \mathrm{C}\right.$ for $30 \mathrm{~s}, 49.5^{\circ} \mathrm{C}$ for $30 \mathrm{~s}$, and $72^{\circ} \mathrm{C}$ for $30 \mathrm{~s}$ ] and a final extension at $72{ }^{\circ} \mathrm{C}$ for $5 \mathrm{~min}$ [11].

Also the COI fragment was amplified using the universal primers below: Forward: 5'-GGAGGATTTGGAAAT TGATTAGTTCC-3' ${ }^{\prime}$ and Reverse: $5^{\prime}$-CCCGGTAAAATT AAAATATAAACTTC-3' [21]. The PCR conditions for COI amplification were set as follows: initial denaturation at $94{ }^{\circ} \mathrm{C}$ for $5 \mathrm{~min}$; followed by 30 cycles of $\left[94{ }^{\circ} \mathrm{C}\right.$ for $30 \mathrm{~s}, 48^{\circ} \mathrm{C}$ for $30 \mathrm{~s}, 72{ }^{\circ} \mathrm{C}$ for $30 \mathrm{~s}$ ] and a final extension at $72{ }^{\circ} \mathrm{C}$ for $7 \mathrm{~min}$.

All the amplicons were sequenced (Bioneer Co., South Korea) and the results were analyzed using BLAST search (https://www.ncbi.nlm.nih.gov).

\section{Phylogenetic analysis}

The phylogeny of the ticks in the present study was deduced by using the Maximum Likelihood method based on the Tamura-Nei model [22]. Initial tree(s) for the heuristic search were obtained automatically by applying Neighbor-Join and BioNJ algorithms to a matrix of pairwise distances estimated using the Maximum Composite Likelihood (MCL) approach, and then selecting the topology with superior log likelihood value. A discrete Gamma distribution was used to model evolutionary rate differences among sites [5 categories $(+\mathrm{G}$, parameter $=2.0513$ for the three genera of Ixodidae ticks and $+\mathrm{G}$, parameter $=0.0500$ for the Dermacentor comparative analysis)]. The MEGA6 software was used for the molecular evolutionary analyses [23]. A bootstrap test including 1000 replicates was performed for each tree and the values (expressed as percentages of 1000 replications) are shown at branch points. 


\section{Results}

In this study, 1229 specimens of Dermacentor marginatus, D. niveus, Hyalomma anatolicum, Rhipicephalus bursa and $R$. sanguineus s.l constituting 11 populations were collected from three different climatic and zoogeographical zones in Iran, and the specimens were investigated using molecular tools (Table1). Phylogenetic analysis of the amplified fragments showed that ITS2 sequences can be used to differentiate the three genera investigated in the present study (Fig. 1).

Similarly, the current protocol using ITS2 could clearly differentiate the two populations of $\mathrm{H}$. anatolicum. Also, the current protocol was able to discriminate the members of Dermacentor from the rest of the genera, but it could not clearly discriminate between the species of this genus (D. marginatus and D. niveus) (Fig. 1a).

For further analysis of the phylogenetic relationship between the different populations of $D$. marginatus and $D$. niveus, the sequences of these species obtained in our study and from other related studies were analyzed (Fig. 1b). The phylogenetic analysis of the different populations of both $D$. marginatus and $D$. niveus revealed clearly that the ITS2 sequences clustered according to location rather than to species such that $D$. marginatus and $D$. niveus collected from Kerman formed their own clade outside the populations of $D$. marginatus and $D$. niveus from Ardebil. Phylogenetic analysis of COI fragment between the sequences obtained from the present study and the sequences obtained from Gene bank for the two species (D. marginatus and D. niveus) showed that these species are in two distinct clades. Also, results of the analysis of the COI fragments suggest that these species can be considered as two distinct species (Fig. 2).

\section{Discussion}

This study is one of the few studies that have focused on the molecular characterization of Ixodidae ticks in Iran. Previously, the ITS2 fragments of different populations of the two species of hard ticks ( $R$. sanguineus s.l and $D$. niveus) have been characterized in Iran $[11,16]$.

Based on the results obtained in our study, which confirm that of earlier studies, it can be safely said that ITS2 is a suitable molecular marker at the genus level for distinguishing the examined genera (Dermacentor, Hyalomma and Rhipicephalus). In addition to the identification at the genus level, the results of this study also indicate that this fragment can be successfully used to discriminate between different species. These results also reinforce the suggestion that ITS2 could be used as a standard DNA barcode in the case of a malfunctioned cytochrome oxidase I (COI) fragment.

The ITS2 marker has been shown to have acceptable results in identifying and examining mosquitoes of different populations within a species, and also, it has shown acceptable ability in the identification of ticks at the species level.

In the recently published lists of ticks, the taxonomic position of some species and the validity of their names have generated controversies among experts $[2,24]$. The species $D$. marginatus and $D$. niveus were previously distinguished solely based on morphological evaluations, but the limitation of the morphological characters is a major concern [16]. Also, comparison of morphological characteristics and examination of the syntype series of $D$. niveus, revealed that $D$. niveus is conspecific with D. marginatus [13]. Similar to this finding, the results obtained in the present study indicate a very high similarity (>98\%) between D. marginatus and D. niveus, based

Table 1 The species, collection sites, climatic zone and the accession numbers of their ITS2 fragment

\begin{tabular}{|c|c|c|c|c|c|}
\hline \multirow[t]{2}{*}{ Species } & \multirow[t]{2}{*}{ Location } & \multirow[t]{2}{*}{ Climatic zone } & \multirow[t]{2}{*}{ Accession no } & \multicolumn{2}{|c|}{ Collected samples } \\
\hline & & & & $\mathbf{n}$ & $\%$ \\
\hline \multirow[t]{3}{*}{ Dermacentor marginatus } & Kerman & Tropical/Desert & KJ004032 & 980 & 79.7 \\
\hline & Meshkin-Shahr & Mediterranean/mountainous & KJ004037 & & \\
\hline & Meshkin-Shahr & Mediterranean/mountainous & KJ004039 & & \\
\hline \multirow[t]{2}{*}{ Dermacentor niveus } & Meshkin-Shahr & Mediterranean/mountainous & KJ004041 & 11 & 0.9 \\
\hline & Kerman & Tropical/desert & KJ004042 & & \\
\hline \multirow[t]{3}{*}{ Rhipicephalus sanguineus s.l } & Tehran & Subtropical/plain & KJ004033 & 18 & 1.5 \\
\hline & Kurdistan & Mediterranean/mountainous & KJ004036 & & \\
\hline & Meshkin-Shahr & Mediterranean/mountainous & KJ004038 & & \\
\hline Rhipicephalus bursa & Meshkin-Shahr & Mediterranean/mountainous & KJ004035 & 195 & 15.9 \\
\hline \multirow[t]{2}{*}{ Hyalomma anatolicum } & Tehran & Subtropical/plain & KJ004034 & 25 & 2.0 \\
\hline & Isfahan & Subtropical/plain & KJ004040 & & \\
\hline Total & & & & 1229 & 100 \\
\hline
\end{tabular}




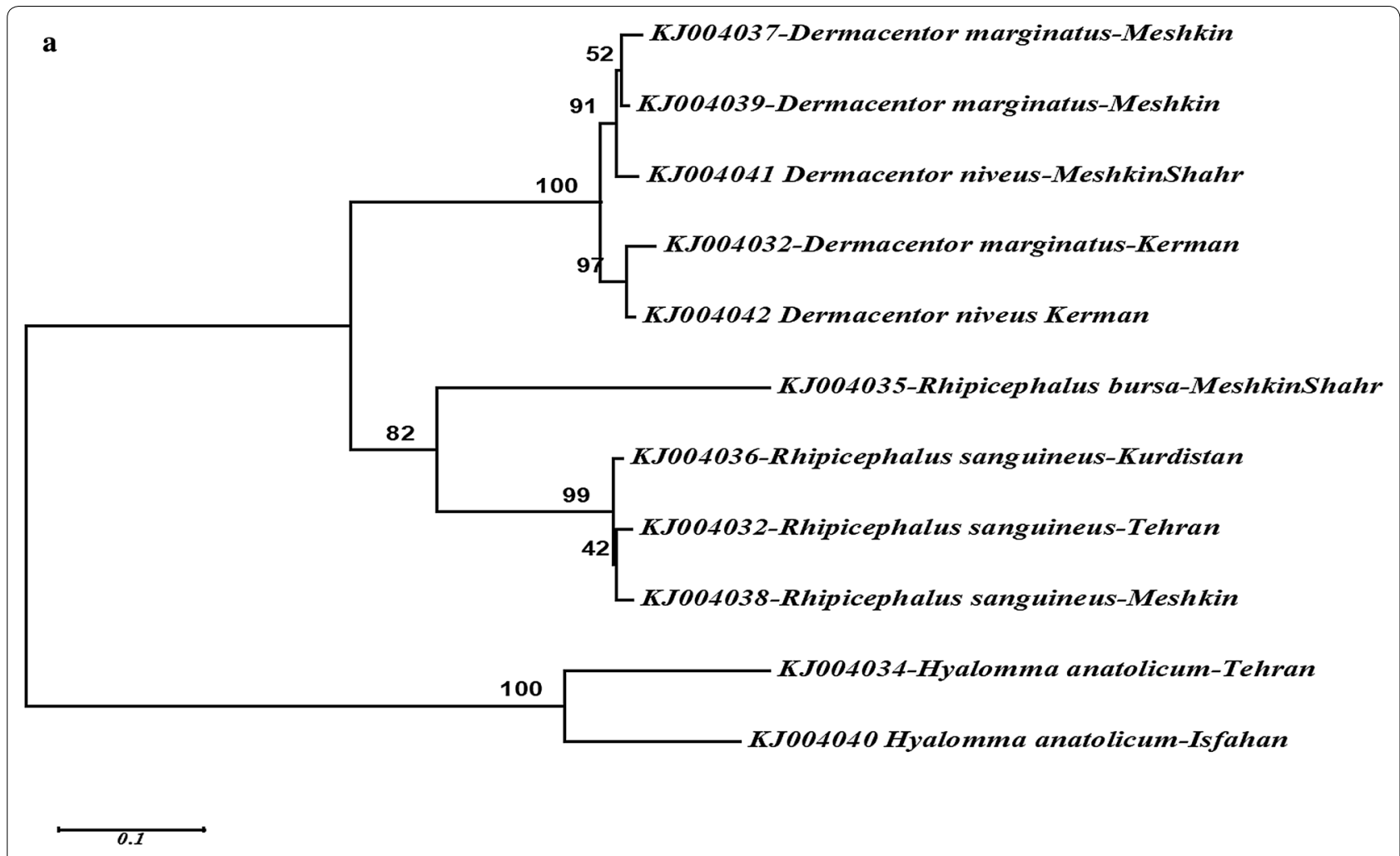

b

KJ004039-Dermacentor marginatus Ardebil

KJ004037-Dermacentor marginatus Ardebil

AJ004041-Dermacentor niveus Ardebil

GQ144706-Dermacentor niveus Iran

FN296269-Dermacentor marginatus Romania

GQ144707-Dermacentor marginatus Iran

KJ004042-Dermacentor niveus kerman

\subsection{5}

Fig. 1 a Phylogenetic relationships based on DNA sequences of 638 bp of ITS2 rDNA region for 11 populations of 5 species belonging to 3 genera of hard ticks. For the species names and abbreviations: D: Dermacentor, R: Rhipicephalus, H: Hyalomma, and Mesh (specimens from Ardebil Province, Meshkin-Shahr, The: Tehran Province, Ker: Kerman Province, Kurd: Kurdistan Province and Isf: Isfahan Province). b Phylogenetic relationship between the different populations of $D$. marginatus and $D$. niveus. The acquired sequences of these species have been indicated with black square for $D$. marginatus and up-pointing triangle for D. niveus 


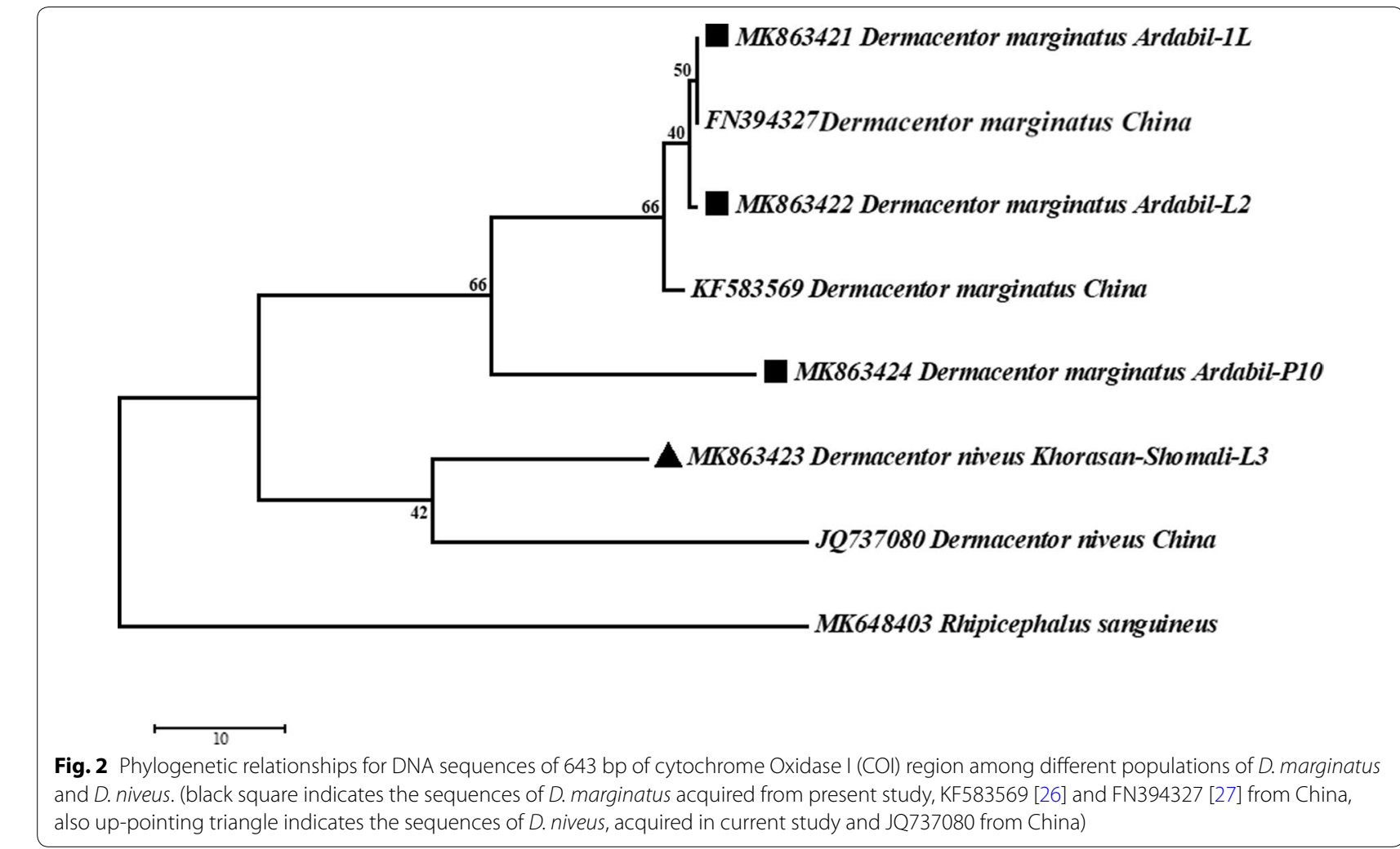

MK863421 Dermacentor marginatus Ardabil-1L

on the analysis of sequence of different populations of both species obtained in our study and from previous studies in Iran [16] and elsewhere [25] (Fig. 2).

On the other hand, analysis of the COI-barcode, which is regarded as a reliable marker for species identification, identified the two species as heterospecific, valid species. This adds to the complexity of identifying the actual status of the taxonomy of these two species.

The results of the analysis of COI fragment in the present study support a recently published data which suggested that $D$. marginatus and $D$. niveus are different species [2].

\section{Limitations}

In the present study, specimens were collected from limited areas. Doing similar studies over a wide geographical area can produce more reliable results. Accordingly, few specimens of $D$. niveus were collected and studied in the present study. Collection of more specimens of this species from more diverse geographical locations can provide a better picture of the genetic diversity of this species.

\section{Supplementary information}

Supplementary information accompanies this paper at https://doi. org/10.1186/s13104-020-05326-5.
Additional file 1: Figure S1. The map of Iran indicating the collection sites of specimens, 1: Ardebil Province (Meshkin-Shahr), 2: Kurdistan Province, 3: Tehran Province, 4: Isfahan Province and 5: Kerman Province. (Base map has been provided by $\mathrm{d}$-maps.com which is freely available).

\section{Abbreviations}

ITS2: Internal transcribed spacer 2; COI: Cytochrome oxidase 1.

\section{Acknowledgements}

The authors would like to thank E. Nemati (Dept. of Environmental Health, SPH, UMSU, Urmia, Iran. H. Hanifian and M. Hasani (Lab. of Hematology, SAM, UMSU, Urmia, Iran) for their kind assistance in experiments. This study was financially supported by the Urmia University of Medical Sciences, Urmia, Iran (Project No. 91-871).

\section{Authors' contributions}

ARC and OT designed and supervised the study, PSA, HE, ZT, FD, HV, MAO and ZR did the field and laboratory activities. MM, SJS, ZZ, HH, FF and MA analyzed the DATA. Also, ARC, PSA, OT, MMB and SJM wrote the draft of the manuscript and ARC and OT finalized the Draft. All authors reviewed the final version of manuscript for publication. All authors read and approved the final manuscript.

\section{Funding}

This study was financially supported by the Urmia University of Medical Sciences (UMSU), Urmia, Iran (Project No. 91-871). The funder had no role in data collection, analysis, interpretation and presentation.

\section{Data availability statement}

The datasets used and/or analyzed during the current study are available from the corresponding author on reasonable request. 
Ethics approval and consent to participate

This project was approved by the Ethics Committee of Urmia University of Medical Sciences.

\section{Consent for publication}

Not applicable.

\section{Competing interests}

The authors declare that they have no competing interests.

\section{Author details}

${ }^{1}$ Social Determinants of Health, Research Center, Urmia University of Medical Sciences, Urmia, Iran. ${ }^{2}$ Department of Medical Entomology and Vector Control, School of Public Health, Urmia University of Medical Sciences, Urmia, Iran. ${ }^{3}$ Department of Medical Entomology and Vector Control, School of Public Health, Tehran University of Medical Sciences, Tehran, Iran. ${ }^{4}$ Department of Infectious Diseases, School of Medicine, Urmia University of Medical Sciences, Urmia, Iran. ${ }^{5}$ Department of Ecology, Swedish University of Agricultural Sciences (SLU), Uppsala, Sweden.

Received: 18 August 2020 Accepted: 4 October 2020

Published online: 09 October 2020

\section{References}

1. Bowman AS, Nuttall PA. Ticks: biology, disease and control. Cambridge: Cambridge University Press; 2008.

2. Guglielmone AA, Robbins RG, Apanaskevich DA, Petney TN, Estrada-Peña A, Horak IG. The hard ticks of the world. New York: Springer; 2014.

3. Cruickshank RH. Molecular markers for the phylogenetics of mites and ticks. Syst Appl Acarol. 2002;7(1):3-14.

4. Navajas M, Fenton B. The application of molecular markers in the study of diversity in acarology: a review. Exp Appl Acarol. 2000;24(10-11):751-74.

5. Hillis DM, Dixon MT. Ribosomal DNA: molecular evolution and phylogenetic inference. Q Rev Biol. 1991;66(4):411-53.

6. Brahma RK, Dixit V, Sangwan AK, Doley R. Identification and characterization of Rhipicephalus (Boophilus) microplus and Haemaphysalis bispinosa ticks (Acari: Ixodidae) of North East India by ITS2 and 16S rDNA sequences and morphological analysis. Exp Appl Acarol. 2014;62(2):253-65.

7. Fukunaga M, Yabuki M, Hamase A, Oliver JH Jr, Nakao M. Molecular phylogenetic analysis of ixodid ticks based on the ribosomal DNA spacer, internal transcribed spacer 2, sequences. J Parasitol. 2000;86(1):38-433.

8. Lempereur L, Geysen D, Madder M. Development and validation of a PCR-RFLP test to identify African Rhipicephalus (Boophilus) ticks. Acta Trop. 2010;114(1):55-8.

9. MCLAIN DK, Wesson DM, Collins FH, Oliver JH. Evolution of the rDNA spacer, ITS 2, in the ticks Ixodes scapularis and I. pacificus (Acari: Ixodidae). Heredity. 1995;75(3):303-19.

10. Zahler M, Gothe R. Evidence for the reproductive isolation of Dermacentor marginatus and Dermacentor reticulatus (Acari: Ixodidae) ticks based on cross-breeding, morphology and molecular studies. Exp Appl Acarol. 1997;21(10):685-96.

11. Abdigoudarzi M, Noureddine R, Seitzer U, Ahmed J. rDNA-ITS2 identification of Hyalomma, Rhipicephalus, Dermacentor and Boophilus spp. (Acari: Ixodidae) collected from different geographical regions of Iran. Adv Stud Biol. 2011;3(5):221-38.
12. Hlinka O, Murrell A, Barker S. Evolution of the secondary structure of the rRNA internal transcribed spacer 2 (ITS2) in hard ticks (Ixodidae, Arthropoda). Heredity. 2002;88(4):275-9.

13. Estrada-Peña A, Estrada-Peña R. Notes on Dermacentor ticks: redescription of $D$. marginatus with the synonymies of $D$. niveus and $D$. daghestaniCus (Acari: Ixodidae). J Med Entomol. 1991;28(1):1-15.

14. Guglielmone AA, Robbins RG, Apanaskevich DA, Petney TN, Estrada-Peña A, Horak IG. Comments on controversial tick (Acari: Ixodida) species names and species described or resurrected from 2003 to 2008. Exp Appl Acarol. 2009;48(4):311.

15. Tekin S, Bursali A, Mutluay N, Keskin A, Dundar E. Crimean-Congo hemorrhagic fever virus in various ixodid tick species from a highly endemic area. Vet Parasitol. 2012;186(3-4):546-52.

16. Moshaverinia A, Shayan P, Nabian S, Rahbari S. Genetic evidence for conspecificity between Dermacentor marginatus and Dermacentor niveus. Parasitol Res. 2009;105(4):1125-32.

17. Ramezani Z, Chavshin AR, Telmadarraiy Z, Edalat H, Dabiri F, Vatandoost H, Zarei Z, Beik-Mohammadi M. Ticks (Acari: Ixodidae) of livestock and their seasonal activities, northwest of Iran. Asian Pac J Trop Dis. 2014;4:S754-S757757.

18. Hoogstraal H. African Ixodoidea Vol. 1. Ticks of the Sudan (with special reference to Equatoria Province and with preliminary reviews of the Genera Boophilus, Margaropus, and Hyalomma). Annapolis: US Navy; 1956.

19. Walker AR. Ticks of domestic animals in Africa: a guide to identification of species. Edinburgh: Bioscience Reports; 2003.

20. Pomerantzev B. Ixodid ticks (Ixodidae). Fauna of USSR, Arachnida, vol. 4 (2). Moscow: USSR Academy of Sciences; 1950.

21. Simon C, Frati F, Beckenbach A, Crespi B, Liu H, Flook P. Evolution, weighting, and phylogenetic utility of mitochondrial gene sequences and a compilation of conserved polymerase chain reaction primers. Ann Entomol Soc Am. 1994;87(6):651-701.

22. Tamura K, Nei M. Estimation of the number of nucleotide substitutions in the control region of mitochondrial DNA in humans and chimpanzees. Mol Biol Evol. 1993;10(3):512-26.

23. Tamura K, Stecher G, Peterson D, Filipski A, Kumar S. MEGA6: molecular evolutionary genetics analysis version 60. Mol Biol Evol. 2013;30(12):2725-9.

24. Filippova N. Taxonomy of ticks of the family Ixodidae (Acarina, Parasitiformes) in the USSR fauna and plans for studying it; 1984.

25. Chitimia L, Lin R, Cosoroaba I, Braila P, Song H, Zhu X. Molecular characterization of hard and soft ticks from Romania by sequences of the internal transcribed spacers of ribosomal DNA. Parasitol Res. 2009;105(4):907-11.

26. Lv J, Wu S, Zhang Y, Chen Y, Feng C, Yuan X, Jia G, Deng J, Wang C, Wang Q. Assessment of four DNA fragments (COI, 16S rDNA, ITS2, 12S rDNA) for species identification of the Ixodida (Acari: Ixodida). Parasites Vectors. 2014;7(1):93.

27. Chitimia L, Lin R-Q, Cosoroaba I, Wu X-Y, Song H-Q, Yuan Z-G, Zhu $X-Q$. Genetic characterization of ticks from southwestern Romania by sequences of mitochondrial cox1 and nad5 genes. Exp Appl Acarol. 2010;52(3):305-11.

\section{Publisher's Note}

Springer Nature remains neutral with regard to jurisdictional claims in published maps and institutional affiliations. 